\title{
Transition of Molecule Orientation during Adsorption of Terephthalic Acid on Rutile $\mathrm{TiO}_{2}(110)$
}

\author{
P. Rahe, ${ }^{\dagger}$ M. Nimmrich, ${ }^{\dagger}$ A. Nefedov, ${ }^{\ddagger}$ M. Naboka, ${ }^{\ddagger}$ Ch. Wöll, ${ }^{\ddagger}$ and A. Kühnle ${ }^{*, \dagger}, \S$ \\ Fachbereich Physik, Universität Osnabrück, Barbarastrasse 7, 49076 Osnabrück, Germany, and Lehrstuhl für \\ Physikalische Chemie I, Ruhr-Universität Bochum, Universitätsstrasse 150, 44780 Bochum, Germany
}

Received: June 3, 2009; Revised Manuscript Received: August 13, 2009

\begin{abstract}
The coverage-dependent mode of adsorption of terephthalic acid $\left[\mathrm{C}_{6} \mathrm{H}_{4}(\mathrm{COOH})_{2}\right.$, TPA] on rutile $\mathrm{TiO}_{2}(110)$ was investigated by means of noncontact atomic force microscopy (NC-AFM) and near-edge X-ray absorption fine structure (NEXAFS) spectroscopy under ultrahigh vacuum conditions at room temperature. Individual molecules are observed to adsorb in an disordered, flat-lying geometry at low coverages up to $\sim 0.3$ monolayer (ML). The molecules are immobile at room temperature, implying a diffusion barrier larger than $0.8 \mathrm{eV}$. This rather high value might be explained by anchoring to surface defect sites. A transition from flat-lying to upright-oriented molecules is revealed by NEXAFS when saturation coverage is achieved. High-resolution NC-AFM images reveal two different structures at coverages between $\sim 0.8$ and $1 \mathrm{ML}$ : (i) a well-ordered $(2 \times 1)$ structure and (ii) a structure of single and paired rows oriented along the [001] crystallographic direction. The latter structure might originate from a pairwise interaction of two neighboring molecules through the top carboxyl groups. Further increase in the exposure results in a saturation of the corresponding signal in the NEXAFS spectra, revealing that the growth of TPA on $\mathrm{TiO}_{2}(110)$ at room temperature is self-limiting.
\end{abstract}

Chemically functionalized surfaces play an important role in many nanotechnological applications. However, only a few attempts have been made so far to create a chemically functionalized metal oxide surface by adsorption of organic molecules, even though metal oxide surfaces provide anisotropy and specific adsorption sites that lead to well-ordered monolayers. Terephthalic acid (TPA) is a versatile molecular linker and has, consequently, received considerable attention with regard to the assembly of three-dimensional self-assembling porous frameworks stabilized by metal-carboxylate bonds, ${ }^{1}$ as well as in two-dimensional supramolecular architectures at surfaces. ${ }^{2-5}$ In particular, TPA/TiO $2(110)$ is a promising system to create an overlayer of upright oriented molecules exposing an organic surface terminated by carboxyl groups. This kind of chemical functionalization could have wide-ranging applications similar to those of carboxyl-terminated SAMs. ${ }^{6}$ It has been previously shown that monolayers of simple monocarboxylic acids form well-ordered $(2 \times 1)$ structures on rutile $\mathrm{TiO}_{2}(110)$ $(1 \times 1)$ surfaces when adsorbed at room temperature. ${ }^{7,8}$ Generally, it is assumed that the carboxylic acid group of the molecule dissociates at room temperature to give carboxylate and hydrogen, although theoretical calculations have yielded rather similar binding energies for the deprotonated and the protonated forms of carboxylic acids on the rutile $\mathrm{TiO}_{2}(110)$ surface. ${ }^{9-11}$ Moreover, it has been discussed whether the dissociated hydrogen plays a role in stabilizing the observed $(2$ $\times 1)$ structure..$^{12,13}$ The negatively charged carboxylate adsorbs on a pair of 5-fold coordinated $\mathrm{Ti}$ atoms in a bridge form with the $\mathrm{O}-\mathrm{C}-\mathrm{O}$ plane aligned along the [001] crystallographic direction. In addition, larger monocarboxylic acids are known to follow this trend upon adsorption onto $\mathrm{TiO}_{2}(110) .{ }^{14}$ The

\footnotetext{
* Corresponding author. E-mail: kuehnle@uni-mainz.de.

$\uparrow$ Universität Osnabrück.

* Ruhr-Universität Bochum.

$\S$ Present address: Institut für Physikalische Chemie, Johannes GutenbergUniversität Mainz, Jakob-Welder-Weg 11, 55099 Mainz, Germany.
}

adsorbate-adsorbate interactions, however, could lead to linkage effects, such as dimerization, that can distort the $(2 \times 1)$ symmetry of the overlayer. Tekiel et al. studied the structure of a complete monolayer of TPA on $\mathrm{TiO}_{2}(110)$ by means of scanning tunneling microscopy (STM) and noncontact atomic force microscopy (NC-AFM). ${ }^{15,16}$ These data indicate that the molecules are oriented upright at a coverage of 1 monolayer (ML). Direct experimental confirmation of the upright orientation of the TPA molecules has not, however, been presented in the literature so far. Moreover, the TPA/TiO $2(110)$ system has been investigated by means of ab initio DFT calculations. ${ }^{9}$ This theoretical work indicates a flat-lying adsorption position of single TPA molecules on a stoichiometric $\mathrm{TiO}_{2}(110)$ surface. The diffusion barrier along the [001] direction has also been calculated, suggesting rather mobile molecules at room temperature.

In this work, we present a coverage-dependent study of TPA adsorption and structure formation on a rutile $\mathrm{TiO}_{2}(110)$ surface. Direct imaging in real space using NC-AFM was complemented by a spatially integrating spectroscopic technique using synchrotron radiation; namely, near edge X-ray absorption fine structure (NEXAFS) spectroscopy. This combined experimental study revealed the presence of disordered, flat-lying molecules at low coverages. Upon increasing the coverage, we observe a transition from flat-lying to upright-oriented molecules, which are found to exhibit two different molecular structures. An ordered $(2 \times 1)$ structure is observed to evolve into a stripelike pattern, which might be due to a pairwise interaction of top carboxyl groups of neighboring TPA molecules. Further increasing the molecular dose leads to a saturation of the resonance signal intensities in NEXAFS, demonstrating that at room temperature, the growth of TPA on this surface is self-limiting and saturates at 1 ML coverage. 


\section{Methods}

Experiments were performed in two separate systems: one ultrahigh vacuum (UHV) system using synchrotron radiation for the NEXAFS experiments and the second UHV system for performing the NC-AFM measurements. Each system, including sample preparation and molecule deposition, is described separately.

For the NEXAFS experiments, the $\mathrm{TiO}_{2}$ crystal (CrysTec $\mathrm{GmbH}$, Berlin, Germany) was introduced into a UHV system with a base pressure better than $10^{-9}$ mbar of the HE-SGM beamline at the Berlin synchrotron radiation facility BESSY II, where all NEXAFS measurements were carried out. Sample cleaning was performed by a few cycles of $\mathrm{Ar}^{+}$sputtering at $T$ $=300 \mathrm{~K}\left(p_{\mathrm{Ar}} \approx 1 \times 10^{-4}\right.$ mbar, $\left.E=800 \mathrm{~V}, I \approx 1 \mu \mathrm{A}\right)$ for $1 \mathrm{~h}$ and annealing at $600 \mathrm{~K}$ for $2 \mathrm{~h}$ in UHV. The cleanliness of the $\mathrm{TiO}_{2}$ surface was checked by comparison with a reference NEXAFS spectrum recorded for a clean $\mathrm{TiO}_{2}$ substrate. The NEXAFS spectra were recorded in the partial electron yield mode (retarding voltage of $150 \mathrm{~V}$ ) with a home-built electron detector based on a double channel plate (Galileo) with an energy resolution of $\sim 0.35 \mathrm{eV}$. The widths of the monochromator slits were set to $200 \mu \mathrm{m}$. A carbon-contaminated gold grid provided a characteristic peak (binding energy (BE) of $284.8 \mathrm{eV}$ ) that was registered simultaneously with each spectrum and served as the reference for photon energy calibration. The raw NEXAFS data were treated using the following procedure: A constant background signal, which was present without illumination, was subtracted from all spectra, then the spectrum of the clean $\mathrm{TiO}_{2}$ crystal was subtracted from the adlayer spectra. The resulting data were divided by a spectrum of a freshly sputtered gold wafer to compensate the energy dependence of the transmission function of the photon flux. Finally, the intensities were normalized to an edge jump of 1 ; i.e., the intensity difference between 278 and $330 \mathrm{eV}$. This processing provides information exclusively on the adsorbed adlayer.

The NC-AFM measurements on the rutile $\mathrm{TiO}_{2}(110)$ surface were performed in a UHV system (base pressure better than $10^{-10}$ mbar) using an NC-AFM (VT AFM 25) from Omicron (Taunusstein, Germany) operated in the frequency modulation (FM) noncontact mode. ${ }^{17} \mathrm{Ar}^{+}$-sputtered silicon cantilevers from NanoWorld (Neuchâtel, Switzerland) with eigenfrequencies of about $300 \mathrm{kHz}$ were excited to amplitudes of $10 \mathrm{~nm}$. Further technical details have been previously described.$^{18}$ The detuning $(\Delta f)$ images presented here are raw data; to all topography $(z)$ images, a plane subtraction was applied unless otherwise noted. The $\mathrm{TiO}_{2}$ samples were crystals of highest available quality (MTI, Richmond, CA). The (110) surfaces were prepared by several cycles of $\mathrm{Ar}^{+}$sputtering at $1 \mathrm{keV}$ for $15 \mathrm{~min}$ and annealing at $1100 \mathrm{~K}$ (measured with a MS09 pyrometer from SensorTherm (Frankfurt am Main, Germany)) for 15 min before molecule deposition. Both molecule deposition and NC-AFM experiments were carried out in UHV keeping the sample at room temperature.

For both experiments, TPA powder from Fluka (Steinheim, Germany) with a purity of better than $99 \%$ was used. The molecular layer was deposited by organic molecular beam deposition (OMBD) using a heated glass crucible. Note that sublimation parameters, such as temperature and electric power, highly depend on the specific design and, therefore, need to be determined for each system separately. Concerning the coverage on $\mathrm{TiO}_{2}(110)$, we define one complete monolayer (1 ML) as one TPA molecule per two $\mathrm{TiO}_{2}(110)$ unit cells; that is, corresponding to the well-known $(2 \times 1)$ structure with a unit cell size of $0.38 \mathrm{~nm}^{2}$, as depicted in Figure 1.

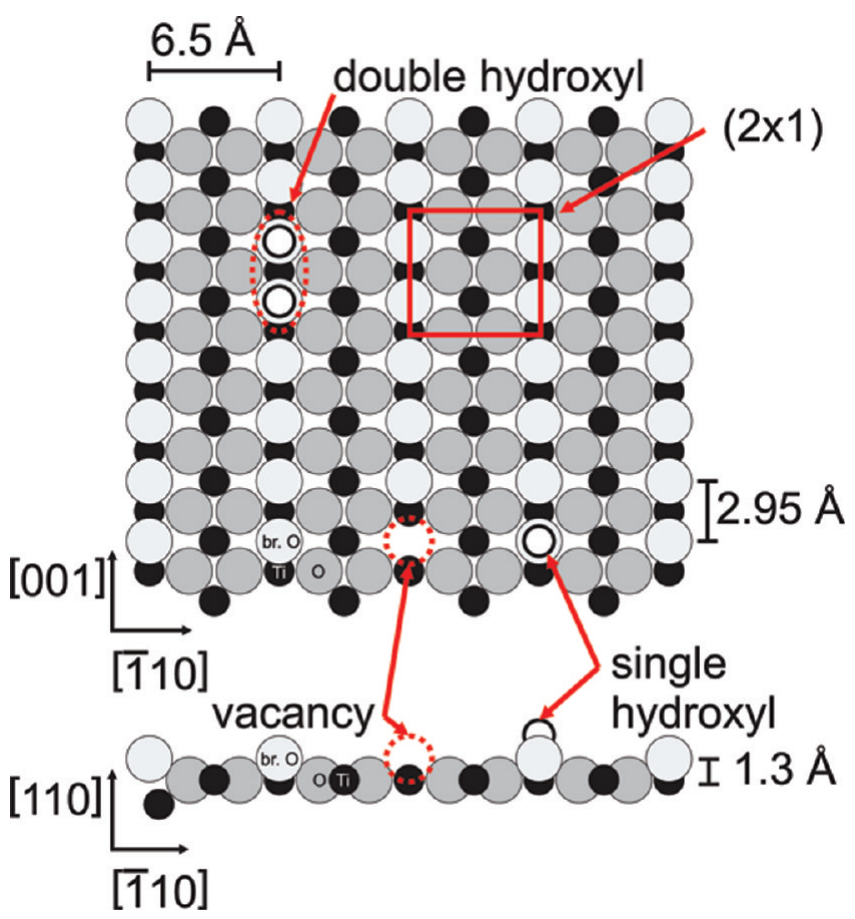

Figure 1. Model of the $\mathrm{TiO}_{2}(110)$ surface. Titanium atoms are shown as small black circles; oxygen atoms, as large gray circles. The bridging oxygen rows are indicated in lighter gray. Additionally, three typical surface defects are shown: oxygen vacancies as well as single and double hydroxyls, all of which are situated in the bridging oxygen rows. The distances indicated are bulk values.

\section{NEXAFS Results}

For the NEXAFS experiments, the deposition rate was measured with a quartz crystal microbalance and was set to 0.07 $\AA / s$ by heating the crucible to $445 \mathrm{~K}$. The distance between the crucible and the $\mathrm{TiO}_{2}$ crystal was about $20 \mathrm{~mm}$.

To determine the dependence of the coverage on the deposition time, we deposited the TPA molecules onto the $\mathrm{TiO}_{2}(110)$ surface in several steps, each lasting 5-15 s. After each deposition step, the sample was transferred from the preparation chamber to the main chamber, and the measurements of the NEXAFS spectra for the $\mathrm{C} \mathrm{K}$ absorption edge as well as a long spectrum including the $\mathrm{C} \mathrm{K}$, Ti $\mathrm{L}_{2,3}$, and $\mathrm{O} \mathrm{K}$ absorption edges at incidence angles of $\theta=20^{\circ}, 55^{\circ}$, and $90^{\circ}$ were performed. By measuring these spectra, it is possible to monitor the increase in the film thickness by taking into account that the jump at the $\mathrm{C} \mathrm{K}$ absorption edge, i.e. the difference of intensities measured before $(E=278 \mathrm{eV})$ and after $(E=330 \mathrm{eV})$ the edge, is proportional to the total number of carbon atoms present on the surface. The lowest curve in Figure 2a presents the NEXAFS spectrum recorded for the pure $\mathrm{TiO}_{2}$ surface. Note the absence of any peaks around the $\mathrm{C} \mathrm{K}$ absorption edge, indicating the presence of a very clean substrate prior to deposition of the TPA molecules. After the TPA deposition, the contribution from the TPA layer appears in the NEXAFS spectra (Figure 2a, middle and topmost curve), while the jump on the Ti absorption edge, that is, the difference of intensities measured at $E=445$ and $480 \mathrm{eV}$, remains the same and can be used for normalization.

In Figure 2b, we present the dependence of the C1s/Ti2p NEXAFS intensity ratio on the deposition time. We can clearly observe a saturation after about $70 \mathrm{~s}$ deposition with the crucible at $445 \mathrm{~K}$, which we assign to $1 \mathrm{ML}$ of TPA. On the basis of the saturation behavior, we can also conclude that a second layer of TPA cannot be grown, or it is very unstable under these conditions. 

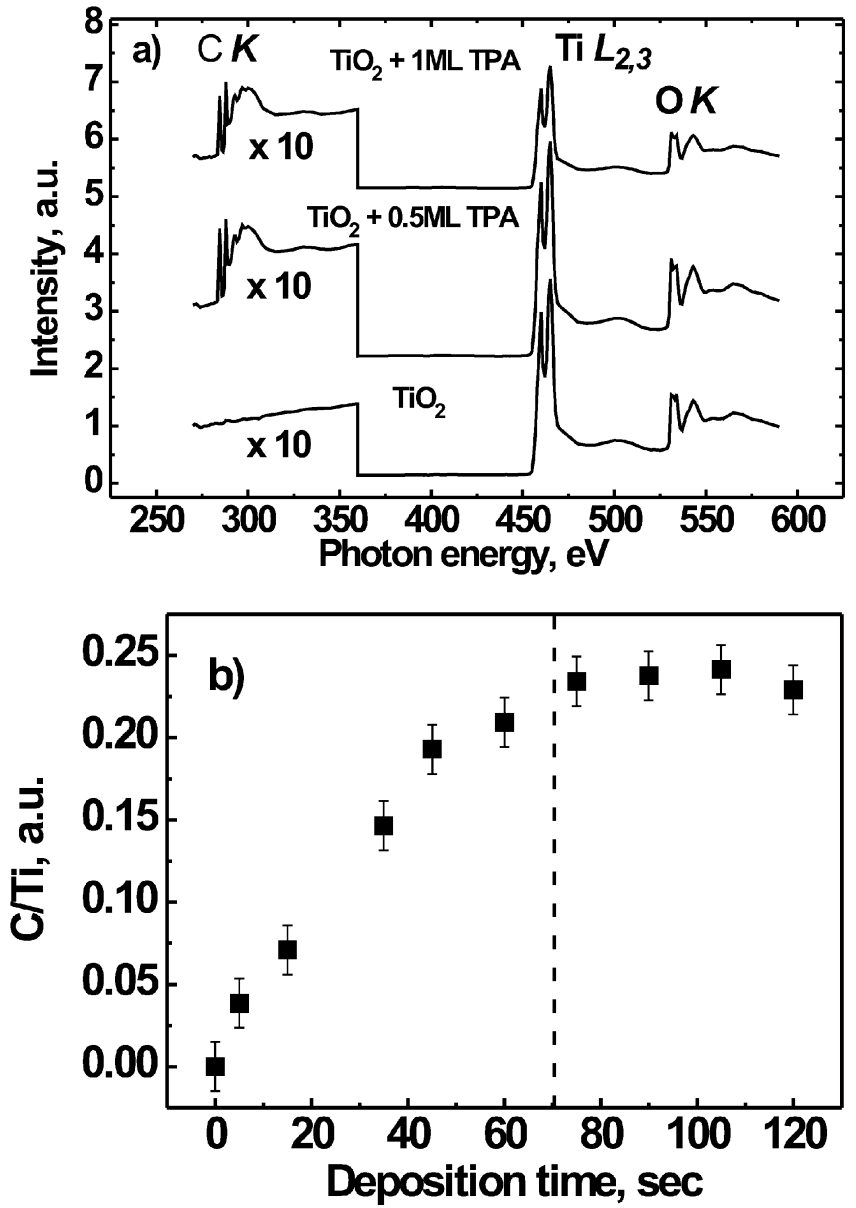

Figure 2. (a) NEXAFS spectra of the TPA/TiO $2(110)$ system for different TPA coverages at an incident angle of $\theta=20^{\circ}$. The signal around the $\mathrm{C} \mathrm{K}$ absorption edge is multiplied by a factor of 10 , and the curves for 0.5 and $1 \mathrm{ML}$ are shifted for clarity. (b) C1s/Ti2p NEXAFS intensity ratio dependence on deposition time. The dashed line corresponds to a deposition time of $1 \mathrm{ML}$.

For a precise determination of the orientation of the TPA molecules, we have determined the dependence of the NEXAFS peak intensities on the photon angle of incidence in the case of the lowest $(0.03-0.07 \mathrm{ML})$ and the highest (1 ML) coverages. To allow for a reliable analysis of the data, the measurements were carried out not only at $\theta=20^{\circ}, 55^{\circ}$ and $90^{\circ}$, but also at $\theta=30^{\circ}$ and $70^{\circ}$ and at different azimuthal orientations of the $\mathrm{TiO}_{2}$ crystal with azimuthal angles $\phi=0^{\circ}$ and $90^{\circ}$ with respect to the [110] direction of the (110) surface.

Figure 3a shows a series of carbon K-edge NEXAFS spectra taken on $\mathrm{TiO}_{2}(110)$ covered with 0.07 ML of TPA molecules, corresponding to a deposition time of $5 \mathrm{~s}$ at a crucible temperature of $445 \mathrm{~K}$. Eight sharp, well-defined peaks are present at the carbon $\mathrm{K}$ between 283 and $291 \mathrm{eV}$ : The first five peaks (at BEs of 284.9, 285.5, 286.8, 288.3, and $290.2 \mathrm{eV}$, respectively) are assigned to $C 1 \mathrm{~s} \rightarrow \pi^{*}$ transitions and the last three (at BEs of 293.2, 297.7, and $301 \mathrm{eV}$, respectively) to C $1 \mathrm{~s} \rightarrow$ $\sigma^{*}$ transitions. ${ }^{19,20}$

The set of spectra in Figure 3 show a clear dependence of the peak intensities on the incident angle, $\theta$. In particular, the pronounced dichroism of the peaks at $E=284.9$ and 285.5 $\mathrm{eV}$, which are related to the phenyl ring, and of the peak at $E$ $=288.3 \mathrm{eV}$ in Figure 3b, which corresponds to the carbonyl group, indicates almost flat-lying molecules. This observation is similar to that reported previously for benzene adsorbed on the same surface. ${ }^{21}$ A numerical fit of the experimental NEXAFS

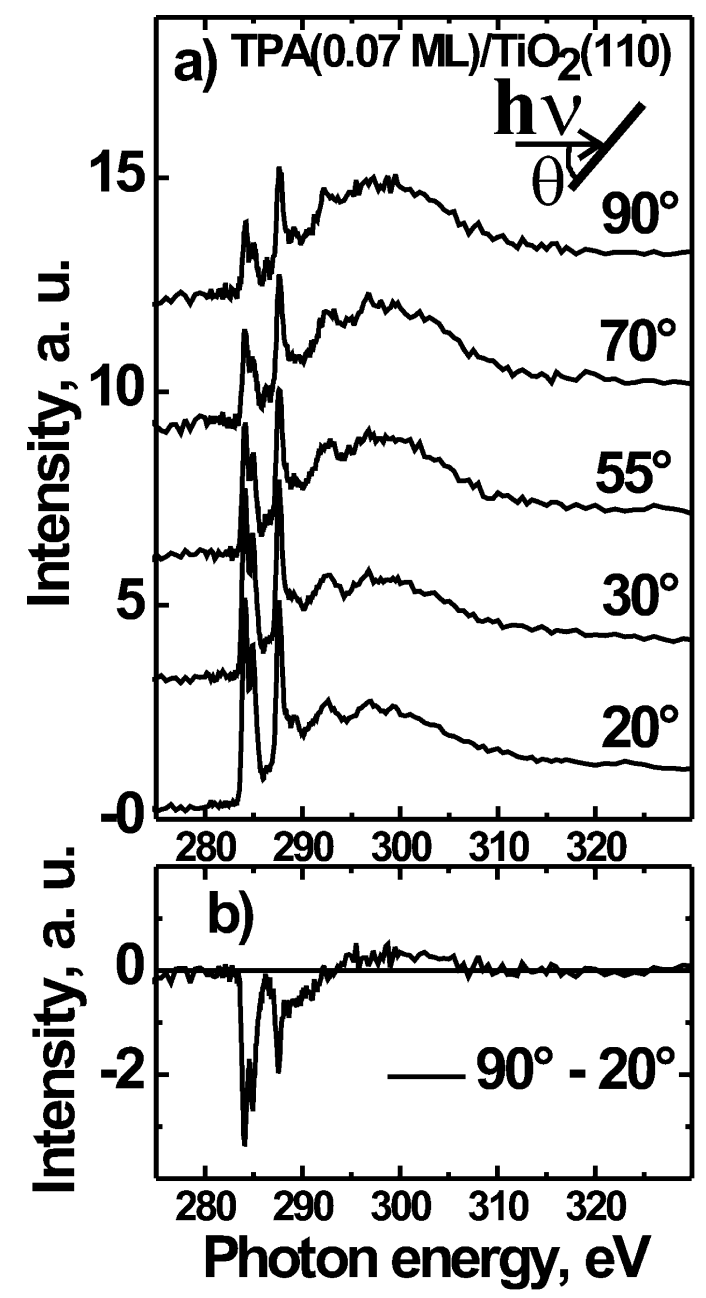

Figure 3. (a) Carbon K NEXAFS spectra for $0.07 \mathrm{ML}$ of TPA coverage measured at different photon incidence angles. (b) Difference of the spectra measured at $\theta=90^{\circ}$ and $20^{\circ}$.

resonance intensities to the theoretical curves for a vector-type $\pi$ orbital $^{22}$ yields an average tilt angle of $18^{\circ}$. For this analysis, we took into account the 2 -fold substrate symmetry and a polarization of the excitation light of $0.82 .{ }^{23}$ The transition moments governing the $\mathrm{C} 1 \mathrm{~s} \rightarrow \pi^{*}$ transitions are oriented perpendicular to the molecular plane of the phenyl ring. Ideally, for TPA molecules in a completely planar adsorption geometry, these peaks should vanish completely for $\theta=90^{\circ}$. However, TPA molecules adsorbed at step edges, defect impurities or dislocation sites might have a more tilted geometry and contribute to the NEXAFS signal, as well. ${ }^{24}$ In addition, a dynamic tilt angle resulting from the thermal occupation of the low energy vibrations of the molecule might also contribute, as was discussed for the case of benzene adsorbed on the same surface. ${ }^{21}$ Thus, we suggest that the adsorption geometry might be even flatter than suggested by the $18^{\circ}$ angle, as deduced from the angular intensity dependence.

The NEXAFS spectra of the 1 ML sample in Figure 4a show the same resonances as observed for the low-coverage $0.07 \mathrm{ML}$ sample in Figure 3a; however, the dependence of the peak intensities on the photon angle of incidence is the opposite. The difference spectrum reproduced in Figure $4 \mathrm{~b}$ demonstrates a positive signal for the $\pi^{*}$ resonances, clear evidence of an upright orientation of the TPA molecules on $\mathrm{TiO}_{2}(110)$. Since the phenyl ring can rotate with respect to the carboxyl group, a random azimuthal orientation of the TPA molecules was 


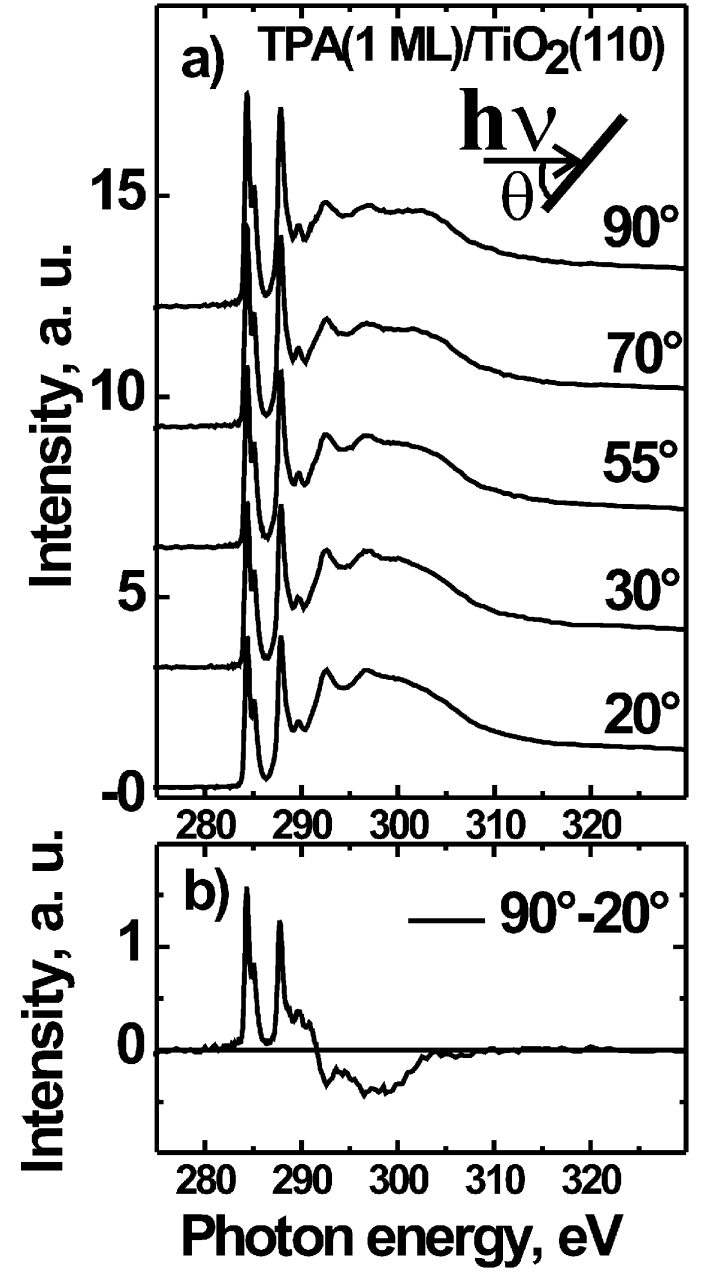

Figure 4. (a) Carbon K NEXAFS spectra for $1 \mathrm{ML}$ of TPA at different photon incidence angles. (b) Difference of the spectra measured at $\theta$ $=90^{\circ}$ and $20^{\circ}$.

assumed. Using this model, we calculated an average tilt angle of the molecules of $70 \pm 10^{\circ}$.

Unfortunately, NEXAFS spectroscopy is rather insensitive to the deprotonation of the carboxylic acid group. Therefore, the question as to whether a (bidentate) carboxylate or a protonated carboxylic acid group binds to the surface cannot be answered on the basis of the present data. Since the acquisition of XPS O 1s data, which are sensitive to the deprotonation, ${ }^{5}$ is hampered by the huge background from the substrate oxygen species, this question has to be left unanswered. Since the dissociated hydrogen atoms might diffuse into the bulk $^{25}$ and lead to a doping of the substrate, in future work, the precise mode of adsorption should be determined using vibrational spectroscopy. ${ }^{26}$

\section{NC-AFM Results}

Also for the NC-AFM experiments, the sublimation characteristics of TPA were investigated with a quartz crystal microbalance (Inficon, East Syracuse, NY) mounted approximately $90 \mathrm{~mm}$ in front of the sublimation cell in place of the $\mathrm{TiO}_{2}$ sample. The temperature range in these experiments (results are presented in Figure 5) was chosen from $\sim 370$ to $\sim 420 \mathrm{~K}$, corresponding to rates as low as $\sim 0.0007 \AA / \mathrm{s}$ and as high as $0.14 \AA$ s. The observed film thickness was different for each measured temperature, but at least $\sim 31 \AA$ up to a maximum of $\sim 700 \AA$. Reflecting the structure formed on $\mathrm{TiO}_{2}(110)$, these

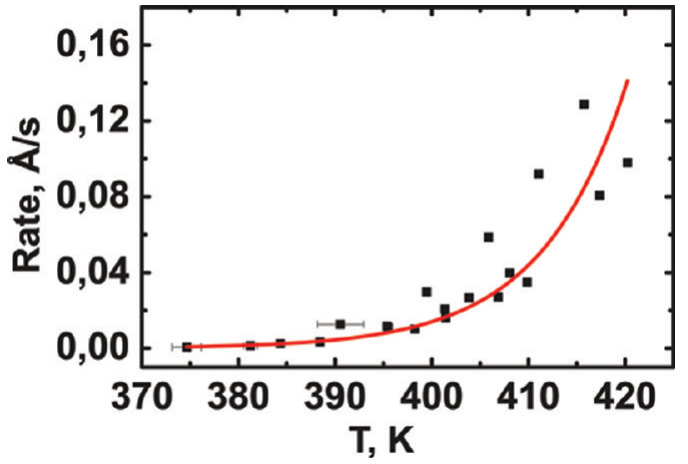

Figure 5. Sublimation characteristics of TPA measured with a quartz crystal microbalance. The temperatures were measured with a thermocouple melted into one end of the crucible and a density of $1.457 \mathrm{~g} / \mathrm{cm}^{3}$ was used to convert to film thickness. ${ }^{27}$

film thicknesses would correspond to coverages on the order of $10-100 \mathrm{ML}$, and therefore, we suggest the film growth is not limited on the crystal sensor in the observed regime of the deposition rate. In contrast, during the NEXAFS experiments, which were performed at a deposition rate of $0.07 \AA /$ s, we observe a limitation as presented in Figure $2 b$. Therefore, we suggest that the substrate-induced order might be responsible for the self-limiting growth revealed by NEXAFS. For the NCAFM experiments, different sublimation temperatures between 382 and $388 \mathrm{~K}$ were used, corresponding to smaller deposition rates between 0.001 and $0.004 \AA$ As. Also in this regime, a second monolayer was not observed.

NC-AFM measures the forces acting between tip and sample, in contrast to STM, in which the local density of states at the
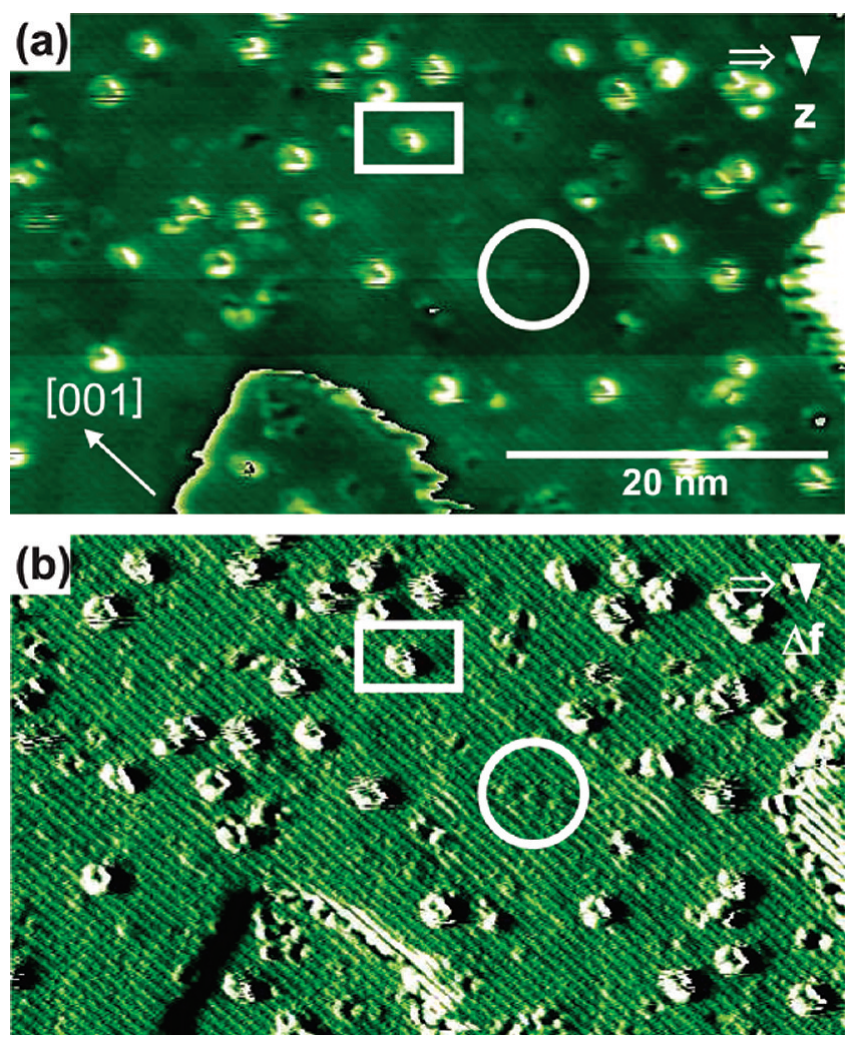

Figure 6. NC-AFM images of TPA on $\mathrm{TiO}_{2}(110)$. (a) Topography and (b) corresponding $\Delta f$ channel. The images are taken in an intermediate constant-height/constant-detuning mode ${ }^{31}$ for tip stability reasons. The bright rows correspond to the titanium rows because linkers are seen in between (some marked by a circle). One single molecule is marked by a rectangle. 

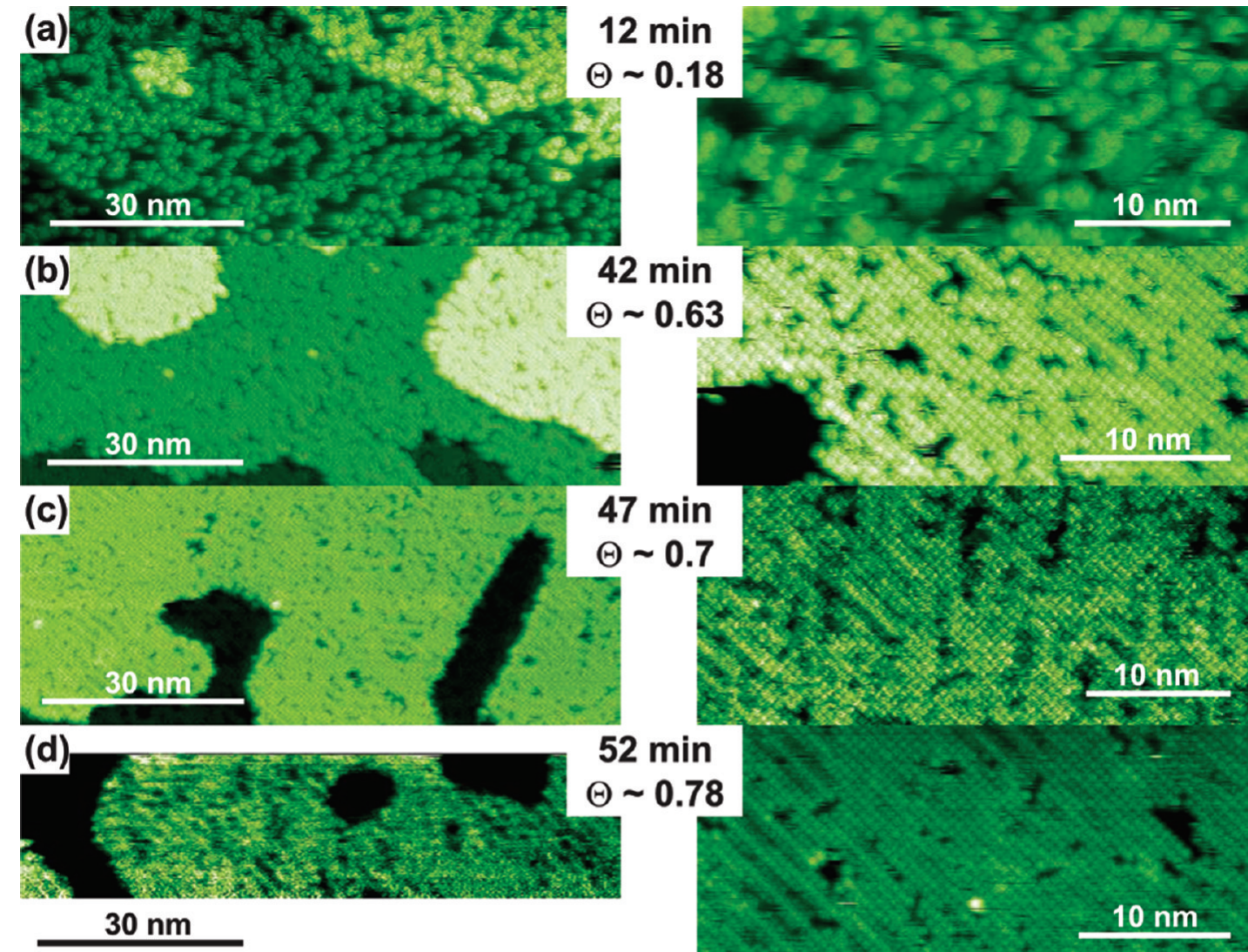

\section{in}
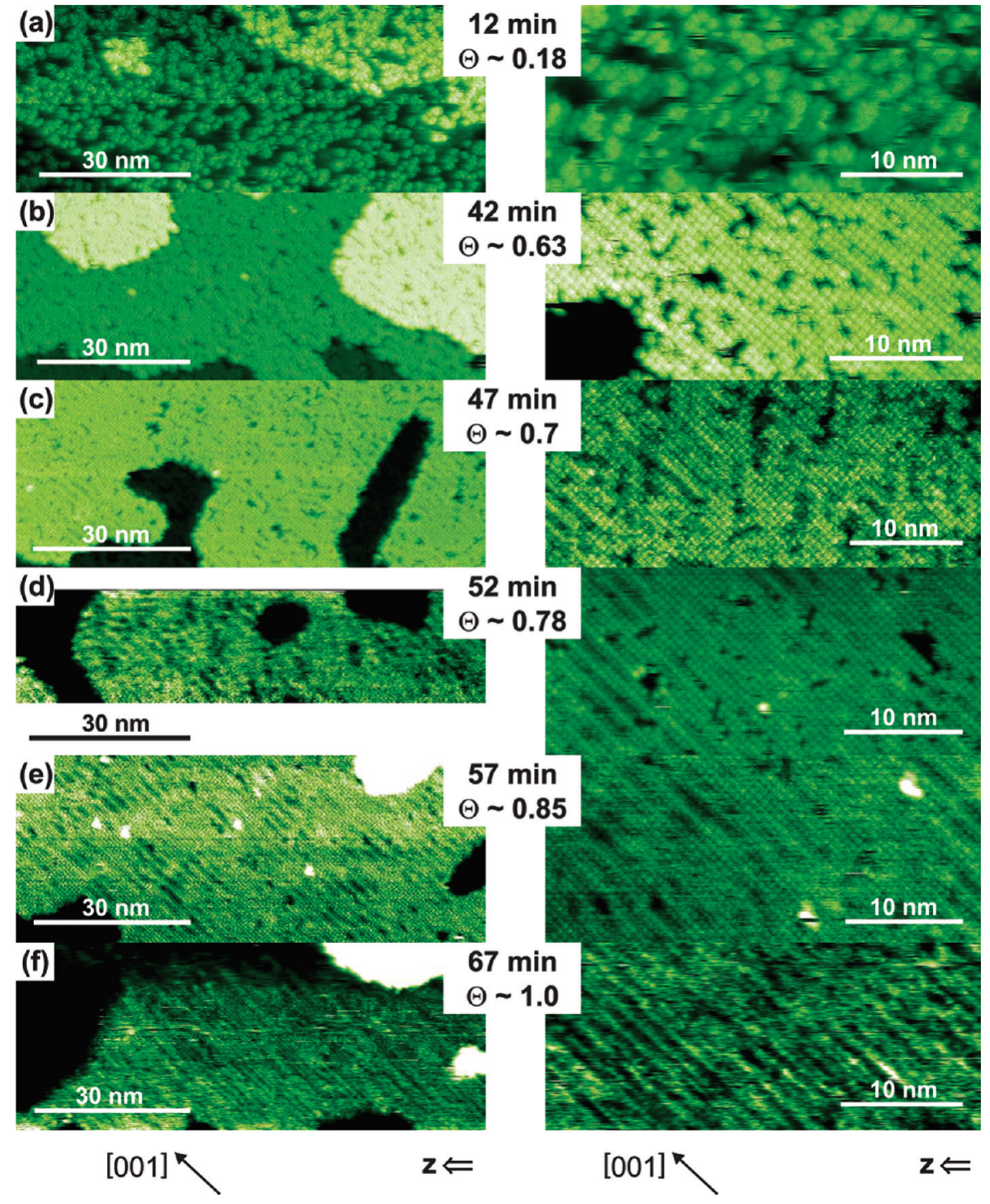

Figure 7. NC-AFM topography images presenting the coverage-dependent structure. The total deposition time and the coverage, $\Theta$, in ML is indicated for each overview (left column) and zoomed (right column) image. All images were taken with the fast scanning direction from right to left.

Fermi level is imaged. Thus, different information is obtained with these two techniques, allowing for gaining complementary information about the investigated system. When imaging $\mathrm{TiO}_{2}(110)$ with NC-AFM, different contrast modes have been reported. ${ }^{28,29}$ Rows along the [001] direction are easily imaged with both AFM and STM. It should be noted, however, that the assignment of these rows to bridging oxygen and in-plane titanium atoms is often ambiguous because it depends on the termination of the AFM tip. Fortunately, the predominant surface defects, mainly consisting of hydroxyls and vacancies, are exclusively found in the bridging oxygen rows and can, thus, serve as a reference for the assignment. ${ }^{30} \mathrm{We}$ can therefore identify the bright substrate rows in Figure 6 as titanium rows: single faint, bright linkers are observed between the bright rows, some marked by a white circle. We attribute these linkers to hydroxyls along the bridging oxygen rows in the [001] direction.

Upon submonolayer deposition of TPA on $\mathrm{TiO}_{2}(110)$, individual molecules are observed on the $\mathrm{TiO}_{2}$ terraces, one of which is marked by a white rectangle in Figure 6. At this coverage of $\sim 0.06 \mathrm{ML}$, neither a long-range ordering nor clustering into islands or decoration of step edges is observed. Instead, individual bright protrusions, some of which exhibit a dark core, are visible and ascribed to single molecules. The position of the molecules relative to the substrate directions could not be identified unambiguously.

Watkins et al. performed ab initio calculations for TPA on a stoichiometric surface. ${ }^{9}$ This theoretical work suggests an adsorption geometry with the phenyl group centered on a 
titanium atom inside the troughs formed by the bridging oxygen rows. This is in agreement with a previous experimental and theoretical study on the adsorption of benzene molecules on the same surface. ${ }^{21}$ For TPA, it has been proposed that such a binding geometry allows the formation of additional bonds between each of the two carboxylic acid groups within the TPA to one in-plane titanium atom and another hydrogen bond to the oxygens in the bridging row. ${ }^{9}$ However, this adsorption geometry might be different in the case of a deprotonated molecule, and so far, it is unclear whether the carboxylic acid groups are deprotonated at this coverage.

Diffusion barriers were calculated in ref 9 for the stoichiometric surface to be 0.39 and $0.22 \mathrm{eV}$ for molecular and deprotonated TPA, respectively. Assuming an attempt frequency of $10^{12} \mathrm{~Hz}$, this would correspond to hopping rates at room temperature of $2.8 \times 10^{6}$ and $2.0 \times 10^{9} \mathrm{~Hz}$, respectively. However, our NC-AFM results indicate a rather stable adsorption of TPA on the surface; motion is observed only very rarely. This is in agreement with prior work of formic acid ${ }^{32}$ and trimethylacetic acid ${ }^{12}$ on $\mathrm{TiO}_{2}(110)$. Both molecules are reported to adsorb rather stably on the surface. Furthermore, the adsorption geometry might significantly differ in the presence of defects on the surface, although this issue has been discussed differently for trimethylacetic acid on rutile $\mathrm{TiO}_{2}(110) .{ }^{12}$ The rather stable adsorption characteristics may, therefore, point toward the fact that TPA favors adsorption positions influenced by surface defects. Furthermore, the adsorption on defect sites might be an explanation for the tilted geometry as observed by the NEXAFS experiments.

The NEXAFS results presented in Figures 3 and 4 provide evidence for different adsorption geometries, depending on the coverage. Corresponding coverage-dependent NC-AFM images are presented in Figure 7. The molecules are deposited successively onto the $\mathrm{TiO}_{2}$ sample while keeping the sublimation cell at $382 \mathrm{~K}$, yielding a sublimation rate of $\sim 0.001 \AA / \mathrm{s}$. A complete monolayer was grown after $\sim 67 \mathrm{~min}$.

These topography images indicate that the deposition of TPA on $\mathrm{TiO}_{2}(110)$ proceeds as follows: At low coverages (Figure 7a) single and clustered molecules are observed with an overall disordered structure. With increasing coverage (Figure 7b), a well-ordered $(2 \times 1)$ structure becomes apparent. In agreement with previous work, ${ }^{15}$ we attribute each bright spot in the AFM images to a single molecule; dark spots appearing within the lines are consequently attributed to missing molecules. Upon further increasing coverage (Figure $7 \mathrm{c}-\mathrm{d}$ ), the number of point defects decreases, and at the same time, a stripelike pattern of dark lines along the [001] direction evolves. The area of these stripelike patches increases with increasing coverage (Figure $7 \mathrm{c}-\mathrm{e}$ ) until the terraces are entirely covered (Figure $7 \mathrm{f}$ ). This structure is assumed to be a $(2 \times 3)$ superstructure.

In Figure 8, taken at a coverage of $\sim 0.9 \mathrm{ML}$, two coexisting structures are present: the $(2 \times 1)$ superstructure and single patches of the striped phase. The overall $(2 \times 1)$ superstructure is corroborated by a two-dimensional Fourier transformation (2D FFT), as shown in the inset of Figure 8: clear spots at $a^{\prime}=$ $(6.1 \pm 0.2) \AA$ and $b^{\prime}=(6.5 \pm 0.2) \AA$ correspond to $\mathrm{a}(2 \times 1)$ superstructure on $\mathrm{TiO}_{2}(110)$, having dimensions of $a=5.91 \AA$ and $b=6.49 \AA$ A. Additionally, few bright species are seen (one marked by a dashed circle in Figure 8). Since the NEXAFS data demonstrate that no second layer of TPA molecules is formed at room temperature, we ascribe these single protrusions to molecules bound to surface impurities. Moreover, single dark spots are observed (one marked by a white triangle), which we assign to single missing TPA molecules in the first monolayer.

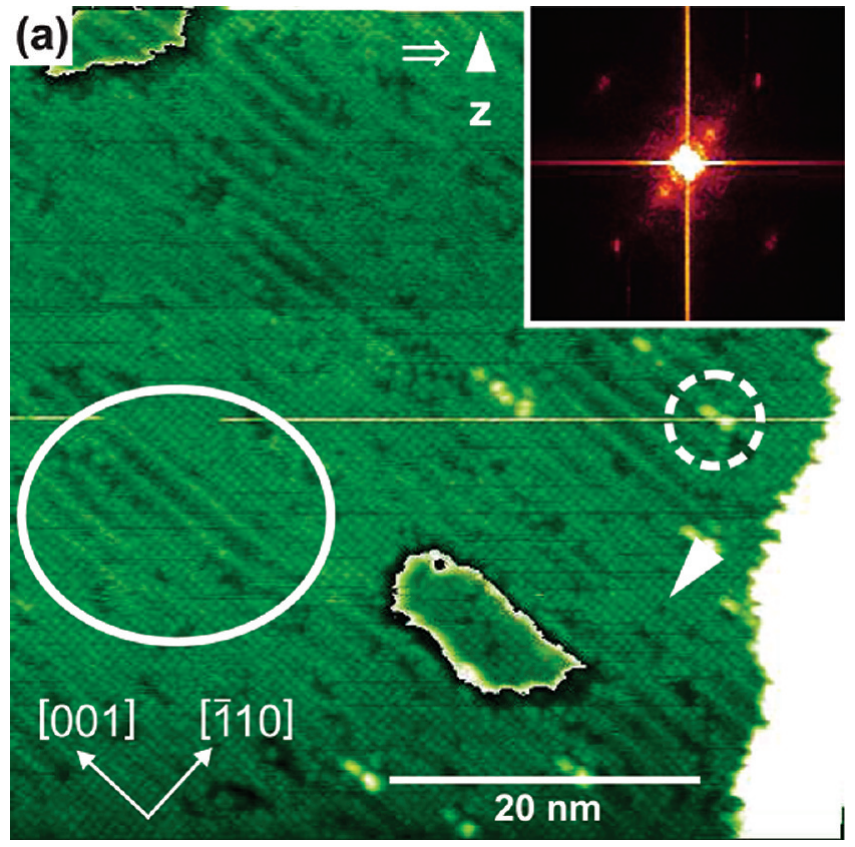

Figure 8. NC-AFM topography image of $\sim 0.9 \mathrm{ML}$ TPA on $\mathrm{TiO}_{2}(110)$. Single defects in the first monolayer are observed, one marked by a white triangle. Molecules on top of the first monolayer are present and ascribed to adsorption due to surface impurities. One is marked by a dashed circle. Furthermore, areas with a striped pattern are seen; one is marked by a solid circle. The inset depicts the 2D FFT of the image, revealing the $(2 \times 1)$ superstructure. Before performing the FFT, the image was properly calibrated as well as corrected for linear drift in the lateral and vertical directions. ${ }^{33}$

The density of these defects decreases when increasing the coverage, as already presented in Figure 7. Interestingly, the above-mentioned striped areas are not observed at coverages of less than $\sim 0.8 \mathrm{ML}$.

The striped area marked in Figure 8 is presented with higher resolution in Figure 9a. This area consists of bright rows along the [001] direction. Between these bright rows, a darker area is found, which might result from two molecular rows pairing together. The apparent height is about $0.3 \AA$ lower as compared to the single bright rows, as shown in the line profile in Figure 9b. Interestingly, within the darker rows, the molecular order along the [001] direction is no longer visible with NC-AFM.

The TPA/TiO 2 (110) system at coverages of 1 ML has been investigated before using STM and NC-AFM. ${ }^{15,16}$ Also in this previous work, a striped structure was observed, which was attributed to alternating single and double molecular rows. ${ }^{15}$ In addition to the striped structure, occasionally, a $(2 \times 1)$ arrangement was imaged and attributed to a different STM tip termination. Note that in this paper, the striped structure exclusively covered entire terraces. On the basis of our coverage-dependent experiments (Figure 7), we conclude that this ostensible discrepancy to our work originates from small differences in the coverage. Whereas in the present experiments, the data were recorded at coverages of around $0.8 \mathrm{ML}$, in the previous work, ${ }^{15}$ only saturated monolayers were studied.

The striped structure has been explained by hydrogen bond formation between the top carboxylic acid groups of two neighboring TPA molecules. ${ }^{15}$ Interestingly, in this model, a single row of unpaired TPA molecules in the [001] direction separates every paired molecular row; this corresponds to a $(2 \times 3)$ superstructure. The driving force governing the 

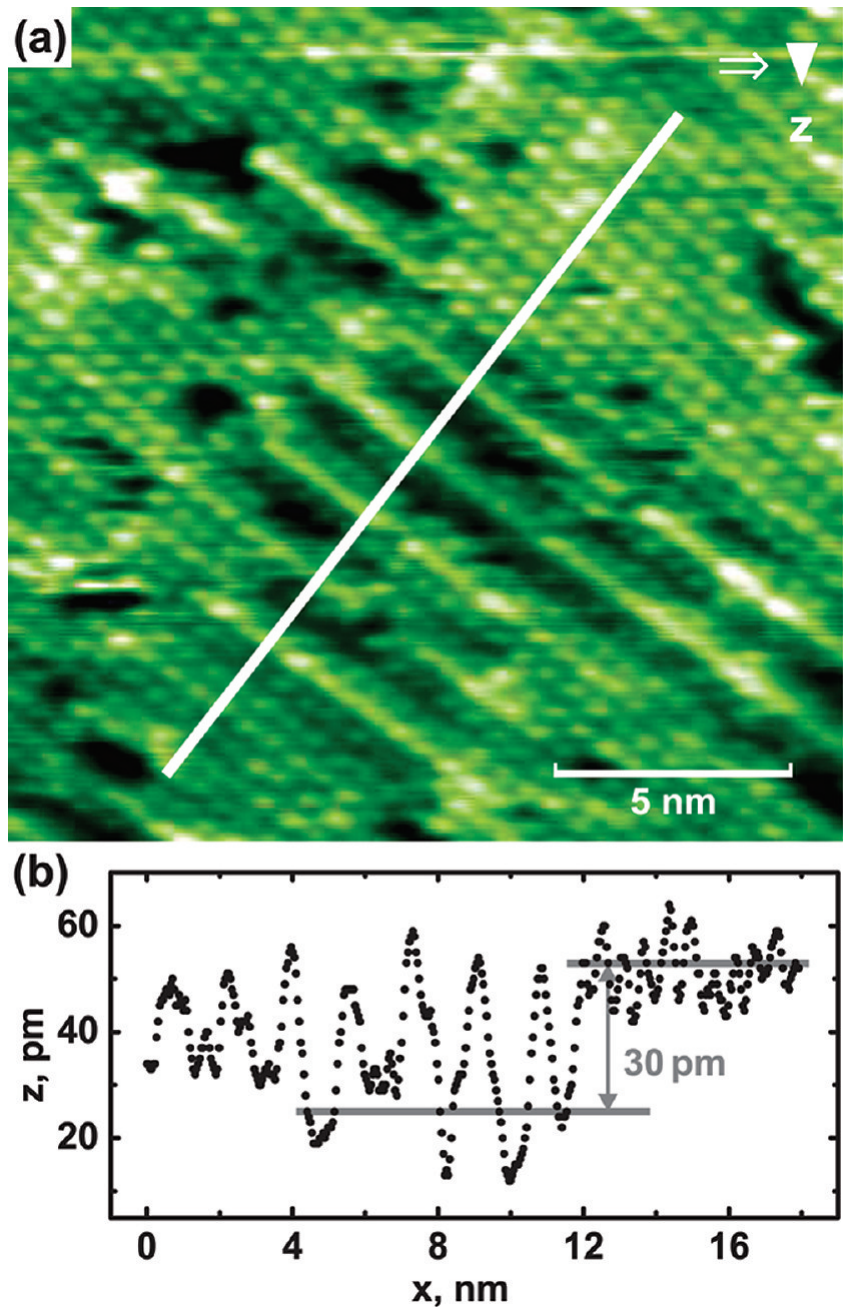

Figure 9. High-resolution image of the striped area, composed of single bright and paired dark rows along the [001] direction. (a) NC-AFM topography image and (b) corresponding line scan at the indicated position. A plane subtraction of second order to compensate for thermal drift and piezo creep and a five pixel mean have been applied. In (b), the vertical difference between bright lines and dark areas of about 0.3 $\AA$ is marked.

formation of these unpaired molecular rows remains unclear on the basis of this simple model. Our results do, however, confirm the existence of regularly spaced unpaired rows in good agreement with the previous findings. ${ }^{15}$

\section{Conclusions}

The adsorption of TPA on the $\mathrm{TiO}_{2}(110)$ was investigated using NC-AFM and NEXAFS spectroscopy over a wide range of coverages, starting at very low values (a few percent of a monolayer) extending to the saturation coverage of $1 \mathrm{ML}$. The NEXAFS data reveal a nearly flat-lying geometry of TPA molecules at low coverages (up to $\sim 0.3 \mathrm{ML}$ ). The molecules are immobile at room temperature, probably as a result of an anchoring to surface defect sites. Upon increasing molecular dose, the coverage saturates at $1 \mathrm{ML}$. When saturation coverage is reached, TPA adsorbs in an upright orientation. NC-AFM images reveal two different structures, depending on the molecule density: (i) a well-organized $(2 \times 1)$ structure at coverages in the range of $\sim 0.3$ to $\sim 0.8 \mathrm{ML}$ and (ii) a stripelike pattern with rows oriented along the [001] direction, resulting in a $(2 \times 3)$ superstructure at coverages in the range of $\sim 0.8$ to $1 \mathrm{ML}$. The total area of regions showing the stripelike pattern increases with increasing coverage. At coverages from $\sim 0.8$ to slightly less than $1 \mathrm{ML}$, the striped pattern coexists with the well-ordered $(2 \times 1)$ structure, whereas for the saturation coverage of $1 \mathrm{ML}$, the entire surface is covered by the striped structure. This striped structure might be explained by a coverage-dependent interaction of the top carboxyl groups of two neighboring TPA molecules. This might also provide an explanation as to why the coverage saturates at $1 \mathrm{ML}$. Because of this bonding within the first monolayer, the binding energy to molecules in the second entire layer is expected to be substantially weakened, thus, suggesting why no second layer was observed in this study.

Acknowledgment. This work was partially supported by the German Research Foundation (DFG) through SFB 558. Travelling costs for synchrotron measurements provided by $\mathrm{BMBF}$ through Grant No. 05ESXBA/5 are gratefully acknowledged. P.R., M.N., and A.K. gratefully acknowledge the support by the DFG through the Emmy Noether program.

\section{References and Notes}

(1) Yaghi, O. M.; O'Keeffe, M.; Ockwig, N. W.; Chae, H. K.; Eddaoudi, M.; Kim, J. Nature 2003, 423, 705-714.

(2) Stepanow, S.; Strunskus, T.; Lingenfelder, M.; Dmitriev, A.; Spillmann, H.; Lin, N.; Barth, J. V.; Wöll, C.; Kern, K. J. Phys. Chem. B 2004, 108, 19392-19397.

(3) Stepanow, S.; Lingenfelder, M.; Dmitriev, A.; Spillmann, H.; Delvigne, E.; Lin, N.; Deng, X.; Cai, C.; Barth, J. V.; Kern, K. Nat. Mater. 2004, 3, 229-233.

(4) Barth, J. V.; Costantini, G.; Kern, K. Nature 2005, 437, 671-679.

(5) Cañas-Ventura, M. E.; Klappenberger, F.; Clair, S.; Pons, S.; Kern, K.; Brune, H.; Strunskus, T.; Wöll, C.; Fasel, R.; Barth, J. V. J. Chem. Phys. 2006, 125, 184710-184718.

(6) Love, J. C.; Estroff, L. A.; Kriebel, J. K.; Nuzzo, R. G.; Whitesides, G. M. Chem. Rev. 2005, 105, 1103-1169.

(7) Diebold, U. Surf. Sci. Rep. 2003, 48, 53-229.

(8) Henderson, M. A.; White, J. M.; Uetsuka, H.; Onishi, H. J. Am. Chem. Soc. 2003, 125, 14974-14975.

(9) Watkins, M.; Trevethan, T.; Sushko, M. L.; Shluger, A. L. J. Phys. Chem. C 2008, 112, 4226-4231.

(10) McGill, P. R.; Idriss, H. Surf. Sci. 2008, 602, 3688-3695.

(11) Bates, S. P.; Kresse, G.; Gillan, M. J. Surf. Sci. 1998, 409, 336349.

(12) Lyubinetsky, I.; Yu, Z. Q.; Henderson, M. A. J. Phys. Chem. C 2007, 111, 4342-4346.

(13) Käckell, P.; Terakura, K. Surf. Sci. 2000, 461, 191-198.

(14) Guo, Q.; Williams, E. M. Surf. Sci. 1999, 433, 322-326.

(15) Tekiel, A.; Prauzner-Bechcicki, J. S.; Godlewski, S.; Budzioch, J.; Szymonski, M. J. Phys. Chem. C 2008, 112, 12606-12609.

(16) Prauzner-Bechcicki, J. S.; Godlewski, S.; Tekiel, A.; Cyganik, P.; Budzioch, J.; Szymonski, M. J. Phys. Chem. C 2009, 113, 9305-9315.

(17) Giessibl, F. J. Rev. Mod. Phys. 2003, 75, 949-983.

(18) Schütte, J.; Bechstein, R.; Rahe, P.; Rohlfing, M.; Langhals, H.; Kühnle, A. Phys. Rev. B 2009, 79, 045428.

(19) Lippitz, A.; Friedrich, J. F.; Unger, W. E. S.; Schertel, A.; Wöll, C. Polymer 1996, 37, 3151-3155.

(20) Okajima, T.; Teramoto, K.; Mitsumoto, R.; Oji, H.; Yamamoto, Y.; Mori, I.; Ishii, H.; Ouchi, Y.; Seki, K. J. Phys. Chem. A 1998, 102, 7093-7099.

(21) Reiss, S.; Krumm, H.; Niklewski, A.; Staemmler, V.; Wöll, C. J. Chem. Phys. 2002, 116, 7704.

(22) Stöhr, J. NEXAFS Spectroscopy; Springer Verlag: Berlin, Heidelberg, New York, 2003.

(23) Tai, Y.; Shaporenko, A.; Eck, W.; Grunze, M.; Zharnikov, M. Appl. Phys. Lett. 2004, 85, 6257.

(24) Mainka, C.; Bagus, P. S.; Schertel, A.; Strunskus, T.; Grunze, M.; Wöll, C. Surf. Sci. 1995, 341, L1055-L1060.

(25) Yin, X. L.; Calatayud, M.; Qiu, H.; Wang, Y.; Birkner, A.; Minot, C.; Wöll, C. ChemPhysChem 2008, 9, 253-256.

(26) Rohmann, C.; Wang, Y. M.; Muhler, M.; Metson, J.; Idriss, H.; Wöll, C. Chem. Phys. Lett. 2008, 460, 10-12.

(27) Bailey, M.; Brown, C. J. Acta Crystallogr. 1967, 22, 387.

(28) Enevoldsen, G. H.; Foster, A. S.; Christensen, M. C.; Lauritsen, J. V.; Besenbacher, F. Phys. Rev. B 2007, 76, 205415-1-205415-14. 
(29) Lauritsen, J. V.; Foster, A. S.; Olesen, G. H.; Christensen, M. C.; Kühnle, A.; Helveg, S.; Rostrup-Nielsen, J. R.; Clausen, B. S.; Reichling, M.; Besenbacher, F. Nanotechnology 2006, 17, 3436-3441.

(30) Wendt, S.; Schaub, R.; Matthiesen, J.; Vestergaard, E. K.; Wahlström, E.; Rasmussen, M. D.; Thostrup, P.; Molina, L. M.; Lægsgaard, E.; Stensgaard, I.; Hammer, B.; Besenbacher, F. Surf. Sci. 2005, 598, 226245 .
(31) Rahe, P.; Bechstein, R.; Schütte, J.; Ostendorf, F.; Kühnle, A. Phys. Rev. B 2008, 77, 195410.

(32) Aizawa, M.; Morikawa, Y.; Namai, Y.; Morikawa, H.; Iwasawa, Y. J. Phys. Chem. B 2005, 109, 18831-18838.

(33) Rahe, P.; Bechstein, R.; Kühnle, A. in preparation, 2009.

JP9052117 